Artículo Original. Enero-Abril 2017; 7(1):48-52. Recibido: 15/01/2017 Aceptado: 07/03/2017.

http://dx.doi.org/10.21929/abavet2017.71.5

\title{
Efecto del consumo de vinagre y una bebida fermentada sobre la calidad de la canal y carne de conejos
}

Effect of the consumption of vinegar and a fermented drink on the quality of the carcass and rabbit meat

\section{Coreno-Hernández Orlando orland_val23@hotmail.com, García-Valencia Saraí saraigarciaval_95@yahoo.com, Ayala-Martínez Maricela ayalam@uaeh.edu.mx, Soto-Simental Sergio sotos@uaeh.edu.mx, Ojeda-Ramírez Deyaniradojeda@uaeh.edu.mx, Zepeda-Bastida Armando*azepeda@uaeh.edu.mx}

Instituto de Ciencias Agropecuarias, Universidad Autónoma del Estado de Hidalgo. México. *Autor responsable y de correspondencia: Zepeda-Bastida Armando Ave. Universidad s/n km 1. Tulancingo, Hidalgo. México.

\section{RESUMEN}

La cunicultura es una actividad que está creciendo a nivel mundial en los últimos tiempos, debido a su fácil manejo, la rapidez con la que se reproducen y la posibilidad de generar animales para su venta 0 autoconsumo. El mantener un sistema digestivo del conejo bajo condiciones apropiadas de $\mathrm{pH}$, hace que el alimento consumido se pueda digerir mejor y los nutrientes estén disponibles para su absorción, además de que el $\mathrm{pH}$ puede ser un factor desencadenante que propicie el crecimiento de microorganismos que puedan afectar la salud del conejo. Aquí evaluamos la calidad de la canal y de la carne de conejo, en animales tratados con vinagre y agua fermentada en agua de bebida, con la finalidad de mejorar el funcionamiento del sistema digestivo, para obtener mejores características de la calidad de la canal y de la carne. Los resultados encontrados mostraron que no existen diferencias significativas si se utiliza vinagre o agua fermentada en la calidad de carne y de la canal en conejos, sólo se observó diferencia significativa en el color, en el valor de $\mathbf{L}(P<0.01)$ en el tratamiento que consumió vinagre; por lo que sería importante utilizar una bebida fermentada con una bacteria ácido-láctica aislada del propio sistema digestivo del conejo y observar su acción sobre la calidad de carne y de la canal.

Palabras clave: Calidad de la carne, producción de conejo, bebida fermentada.

\begin{abstract}
Rabbit breeding is an activity that is growing worldwide in recent times, due to its easy handling, the speed with which they reproduce and the possibility of generating animals for sale or self-consumption. Keeping rabbit's digestive system under appropriate $\mathrm{pH}$ conditions means that the food consumed can be better digested and nutrients are available for absorption, and $\mathrm{pH}$ can be a triggering factor that encourages the growth of microorganisms that may affect rabbits' health. Here we evaluated the quality of the carcass and rabbit meat in animals treated with vinegar and fermented water in consumption water, in order to improve the functioning of the digestive system, to obtain better quality characteristics of the carcass and the meat. The results showed that there are no significant differences when using vinegar or fermented water in the meat quality and the carcass in rabbits, only a significant difference in color was observed in the value of $L$ $(\mathrm{P}<0.01)$ in the treatment which consumed vinegar; so it would be important to use a fermented drink with an acid-lactic bacterium isolated from the rabbit's own digestive system and observe its action on the quality of meat and the carcass.
\end{abstract}

Keywords: Meat quality, rabbit production, fermented beverage. 


\section{INTRODUCCIÓN}

La producción de conejo en México está localizada principalmente en los estados del centro del país, como Hidalgo, Puebla, Tlaxcala, Estado de México y Guanajuato. Aún y cuando la composición nutricional de la carne de conejo es buena, el consumo per cápita en México es bajo, de acuerdo con Armada (2016) puede variar de 38 a 134 g entre 2008 y 2009. Este bajo consumo puede ser derivado del bajo interés que muestran las instituciones públicas para fomentar la producción de conejos; además de la poca difusión que se hace sobre el consumo de esta carne (Olivares et al., 2015); a pesar de ser considerada una carne que tiene diversas ventajas con relación a otros tipos de carne, principalmente desde el punto de vista nutricional; ya que se ha observado que tiene un balance adecuado de ácidos grasos, proteína, vitaminas y minerales, bajo en colesterol y sodio, para el consumo humano (Para, 2015).

El mantener un sistema digestivo del conejo bajo condiciones apropiadas de $\mathrm{pH}$ dependiendo de la región del mismo, hace que el alimento consumido se pueda digerir mejor y los nutrientes estén disponibles para su absorción (Carabaño y Piquer, 1998); además de que el $\mathrm{pH}$ puede ser un factor desencadenante que propicie el crecimiento de microorganismos que puedan afectar la salud del conejo. El tener una fuente apropiada de nutrientes y un sistema digestivo sano, permite que el conejo sea más productivo en términos de ganancia diaria de peso, consumo de alimento, conversión alimenticia; lo cual puede conllevar a tener una mejor calidad de la canal y de la carne (Xiccato, 1999). Por tal motivo es necesario desarrollar investigaciones que fortalezcan el manejo productivo, nutricional y de calidad de la carne de esta especie, aunado a ello implementar estrategias que permitan mejorar el rendimiento económico para los productores de conejo.

El objetivo del presente estudio fue el de evaluar la calidad de la canal y de la carne de conejo, en animales tratados con vinagre y agua fermentada en agua de bebida; con la finalidad de mejorar el funcionamiento del sistema digestivo, para obtener mejores características de la calidad de la canal y de la carne.

Se utilizaron seis conejos de la cruza Chinchilla $\times$ Nueva Zelanda $\times$ California, destetados a los $35 \mathrm{~d}$ de edad, los cuales fueron distribuidos en dos tratamientos; T1 consumió 20 $\mathrm{ml}$ de vinagre comercial ( $5 \%$ de ácido acético) en $1 \mathrm{~L}$ de agua ( $\mathrm{pH} 4.6)$ y T2 consumió 80 $\mathrm{ml}$ de leche fermentada comercial, también en $1 \mathrm{~L}$ de agua (pH 6.4). Los conejos fueron alimentados ad libitum con alimento comercial durante el periodo de engorda (30 d). El consumo de alimento y agua fueron medidos diariamente, los animales fueron pesados una vez por semana.

Después de la engorda los animales fueron transportados al Taller de Cárnicos del Instituto de Ciencias Agropecuarias, donde fueron sacrificados de acuerdo a la NOM- 
033-SAG/ZOO 2015, la canal fue refrigerada en una cámara a $4^{\circ} \mathrm{C}$ por $24 \mathrm{~h}$, para posteriormente ser diseccionada de acuerdo a las recomendaciones de Blasco y Ouhayoun (1996); para lo cual se pesó: la canal caliente, piel, patas, vísceras, canal fría, cabeza, parte anterior, media y posterior, además de piernas.

En lo que se refiere a la calidad de la carne, se midió en el lomo, pH (Oakton), color (Microoptix S560, I-Lab); y un análisis de perfil de textura en carne cocinada (Brookfield CT3). Para el análisis estadístico se empleó un diseño completamente al azar con dos tratamientos, y cuando se encontraron diferencias se utilizó una prueba de Tukey.

\section{RESULTADOS Y DISCUSIÓN}

La inclusión de vinagre o de bebida fermentada en el agua de bebida de los conejos, no presenta un efecto sobre los parámetros productivos durante la engorda (Cuadro 1); sin embargo se puede observar una tendencia a mejorar los parámetros productivos en el tratamiento con bebida fermentada, como es el caso de la ganancia de peso total. Musa et al., (2009) observaron que al adicionar enzimas y probióticos, se mejora la ganancia de peso y la conversión alimenticia. Así como Sharma et al., (2016), quienes indican que al incluir en la engorda de conejos probióticos se mejoran los parámetros productivos.

\begin{tabular}{|c|c|c|}
\hline \multirow[b]{2}{*}{ Parámetros productivos } & \multicolumn{2}{|c|}{ Tratamiento } \\
\hline & $\begin{array}{c}\text { Vinagre } \\
20 \mathrm{~mL} \mathrm{~L}^{-1}\end{array}$ & $\begin{array}{l}\text { Bebida fermentada } \\
\qquad 80 \mathrm{~mL} \mathrm{~L}^{-1}\end{array}$ \\
\hline PV inicial $(g)$ & 520.10 & 487.03 \\
\hline PV final $(g)$ & 1950.00 & 2103.3 \\
\hline Ganancia total (g) & 1429.90 & 1616.3 \\
\hline GDP total $(\mathrm{g} / \mathrm{d})$ & 34.88 & 39.420 \\
\hline Rendimiento de la canal caliente (\%) & 56.101 & 55.357 \\
\hline Vísceras (\%) & 21.51 & 21.8015 \\
\hline Piel (\%) & 13.2825 & 14.9086 \\
\hline Patas (\%) & 3.4951 & 2.9456 \\
\hline
\end{tabular}

GDP=Ganancia diaria de peso

$\mathrm{PV}=$ Peso vivo

Cuadro 1. Parámetros productivos durante la engorda de conejos que se les ofreció vinagre y una bebida fermentada en el agua.

En el Cuadro 2 se muestran los datos de la evaluación de la canal de conejos alimentados con vinagre y una bebida fermentada; los resultados encontrados mostraron que no existen diferencias significativas en la calidad de la canal; sin embargo los conejos tratados con bebida fermentada, mostraron pesos mayores; lo cual podría ser debido a que la bebida fermentada contribuye en el balance de la flora microbiana del sistema digestivo del conejo y hace que éste sea más eficiente en la digestión de los alimentos, 
para mejorar la absorción de nutrientes; lo cual se traduce en mejor calidad de la canal; tal y como mencionan Sharma et al., (2016), quienes indican que el porcentaje de rendimiento de la canal de conejos se ve afectada por el uso de probióticos.

\begin{tabular}{lcc}
\hline \multirow{2}{*}{ Parámetros } & \multicolumn{2}{c}{ Tratamiento } \\
\cline { 2 - 3 } & $\begin{array}{c}\text { Vinagre } \\
20 \mathrm{~mL} \mathrm{~L}^{-1}\end{array}$ & $\begin{array}{c}\text { Bebida fermentada } \\
80 \mathrm{~mL} \mathrm{~L}^{-1}\end{array}$ \\
\hline Peso de la canal fría $(\mathrm{g})$ & 1078 & 1133 \\
Peso de la cabeza $(\mathrm{g})$ & 133 & 118 \\
Peso parte anterior $(\mathrm{g})$ & 296 & 284 \\
Peso parte media $(\mathrm{g})$ & 77 & 93 \\
Peso parte posterior $(\mathrm{g})$ & 210 & 233 \\
Peso de las piernas $(\mathrm{g})$ & 346 & 364 \\
Peso de los riñones $(\mathrm{g})$ & 35 & 34 \\
\hline
\end{tabular}

Cuadro 2. Calidad de la canal de conejos que se les ofreció vinagre y una bebida fermentada en el agua.

Como parte de la evaluación de la calidad de la carne, en lo que se refiere al color, el valor de $\mathbf{L}$ fue significativamente mayor $(\mathrm{P}<0.01)$ en el tratamiento que consumió vinagre (Cuadro 3). Los demás parámetros no mostraron diferencias significativas; sin embargo la carne de conejos alimentados con la bebida fermentada tiene un valor de dureza menor.

Existe poca información sobre el efecto que tiene, tanto el ácido acético como probióticos, sobre la calidad de la carne, principalmente en el color de la carne y la textura.

\begin{tabular}{|c|c|c|}
\hline \multirow[b]{2}{*}{ Parámetros } & \multicolumn{2}{|c|}{ Tratamiento } \\
\hline & $\begin{array}{c}\text { Vinagre } \\
20 \mathrm{~mL} \mathrm{~L}^{-1}\end{array}$ & $\begin{array}{l}\text { Bebida fermentada } \\
\quad 80 \mathrm{~mL} \mathrm{~L}^{-1}\end{array}$ \\
\hline $\mathrm{pH}$ & 6.14 & 6.18 \\
\hline Dureza (gf) & 1638.80 & 1396.50 \\
\hline Adhesividad & 0.07 & 0.04 \\
\hline Resiliencia & 0.21 & 0.22 \\
\hline Cohesividad & 0.53 & 0.58 \\
\hline Elasticidad & 2.74 & 2.798 \\
\hline$L^{*}$ & $50.72^{\mathrm{a}}$ & $48.316^{b}$ \\
\hline$a^{*}$ & 0.73 & 0.617 \\
\hline$b^{*}$ & -5.28 & -5.711 \\
\hline
\end{tabular}

Cuadro 3. Calidad de la carne de conejos que se les ofreció vinagre y una bebida fermentada en el agua. 


\section{CONCLUSIÓN}

Los resultados encontrados mostraron que el uso de vinagre y/o bebida fermentada, como agua de bebida en la producción de conejos, fue similar en los parámetros productivos, calidad de la canal y de la carne; excepto para el parámetro de color (L), el cual fue mayor en los animales que consumieron vinagre.

\section{IMPLICACIONES}

La inclusión de una bebida fermentada en el agua de bebida de los conejos, puede ser benéfica para mejorar la calidad de la canal y de la carne; sin embargo sería importante utilizar una bebida fermentada con una bacteria ácido-láctica aislada del propio sistema digestivo del conejo.

\section{LITERATURA CITADA}

ARMADA ER. 2016. La explotación cunícola en México, una revisión a través del VIII Censo Agrícola, Ganadero y 2007. http://www.ancum.com.mx/web/pdfs/Organizacion\%20de\%20productores/L A\%20EXPLOTACION\%20CUNICOLA\%20EN\%20MEXICO.pdf.

BLASCO A, Ouhayoun J. 1996. Harmonization of criteria and terminology in rabbit meat research. World Rabbit Science, 4:93-99.

CARABAÑO K, Piquer J. 1998. The digestive system of the rabbit. In: The nutrition of the rabbit. Ed. C. de Blas and J. Wiseman. CABI Publishing. London, UK.

MUSA HH, Wu SL, Zhu CH, Seri HI y Zhu GQ. 2009. The Potential Benefits of Probiotics in Animal Production and Health. Journal of Animal and Veterinary Advances, 8(2): 313321.

OLIVARES PR, Gómez CMÁ., Schewentesius RR y Carrera CB. 2009. Alternativas a la producción y mercadeo para la carne de conejo en Tlaxcala, México. Región y Sociedad. Septiembre-Diciembre, XXI (46), pp. 191-207.

PARA PA, Ganguly S, Wakchaure R, Sharma R, Mahajan T, y Praveen PK. 2015. Rabbit meat has the potential of being a possible alternative to other meats as a protein source: A brief review. Int J Phar Biomedi Res. 2: 17-19.

SHARMA KG, Vidyarthi VK, Archana K, Zuyie R. 2016. Probiotic supplementation in the diet of rabbits - A review. Livestock Research International. 4:1-10.

XICCATO G. 1999. Feeding and meat quality in rabbits: a review. World Rabbit Science. 7:75-86.

\section{AGRADECIMIENTOS}

El presente trabajo fue financiado por SEP-PROMEP con número de asignación DSA/103.5/16/10281. 